\title{
A minimum principle for plurisubharmonic functions
}

\author{
Ahmed Zeriahi
}

\begin{abstract}
The main goal of this work is to give new and precise generalizations to various classes of plurisubharmonic functions of the classical minimum modulus principle for holomorphic functions of one complex variable, in the spirit of the famous lemma of Cartan-Boutroux. As an application we obtain precise estimates on the size of "plurisubharmonic lemniscates" in terms of appropriate Hausdorff contents.
\end{abstract}

AMS 2000 Mathematical subject classification: 31C10, 31C15, 32F05, 32F99, 32U05, 32U99.

\section{Introduction}

In Complex Analysis of one variable, besides the classical maximum principle, there is another principle that is less well known, but just as important and somewhat more subtil. It consists in giving a precise lower bound for the modulus of a holomorphic function (normalized in a suitable way) on a given open disc at all points of a smaller disc, except those which belong to an exceptionnal subset containing its zeros, in terms of its maximum on the given disc; the size of the exceptionnal set should be precisely estimated in terms of capacity or one dimensionnal Hausdorff content. This is called the minimum modulus principle for holomorphic functions (see [26], [35]).

This principle plays an important role in many problems involving rationnal or meromorphic functions which may have many poles in a given domain and then it's desirable to find an upper bound of such function, which remains to control precisely the subset where the denominator of the function is small.

Classically in one complex variable, this can be done using the well know Cartan-Boutroux' lemma, which gives a precise uniform estimate of the size of (monic) polynomial lemniscates in terms of one dimensionnal Hausdorff content.

This kind of estimates can be applied in Nevalinna theory for the study of the growth of holomorphic functions (see [10], [28]) as well as in Padé approximation when studing the convergence of rationnal Padé approximants 
of meromorphic functions (see [13], [18]) and also in Harmonic Analysis and PDE's (see [16], [9]).

Uniform estimates on the size of sublevel sets of some classes of plurisubharmonic functions, called plurisubharmonic lemniscates, have been obtained in our earlier papers (see [39],[40], [8]).

The main purpose of the present paper is to give precise and new various generalizations of the Cartan-Boutroux' lemma to logarithmic potentials in $\mathbb{C}^{n}$ as well as a general version of the minimum principle for arbitrary plurisubharmonic functions on euclidean balls in $\mathbb{C}^{n}$.

From the version of the minimum principle for logarithmic potentials in $\mathbb{C}^{n}$, we deduce a new comparison inequality between appropriate Hausdorff contents and the classical logarithmic capacity in $\mathbb{C}^{n}$.

The method used here, known as the method of "excluding balls" ("boules d'exclusion" in french), seems to be quite classical in real potential theory when estimating integral potentials but surprinsingly, it has never been used in our context although it has been used in a different context by Avanissian ([4]).

It turns out that the same method allows us to obtain a quite general lower estimate for plurisubharmonic functions on the unit ball, which implies a sort of "three-circle minimum principle" for plurisubharmonic functions, which can be seen as the dual counterpart of Hadamard three-circle inequalities.

Actually, the same method can be also applied on a compact Kähler manifold to give a minimum principle for quasiplurisubharmonic functions on the manifold.

\section{The Cartan-Boutroux lemma and the minimum principle}

Let us first recall the famous classical lemma of Cartan-Boutroux (see [10]). Let $P(z)$ be a monic polynomial of one complex variable of degree $d \geq 1$. For any given $\varepsilon>0$, consider the polynomial $\varepsilon$-lemniscate of $P$ defined by $E(P ; \varepsilon):=\left\{z \in \mathbb{C} ;|P(z)| \leq \varepsilon^{d}\right\}$.

Then there exists a finite covering of $E(P ; \varepsilon)$ by $d$ open discs with radii $\left(r_{j}\right)_{1 \leq j \leq d}$ satisfying the estimate

$$
\sum_{1 \leq j \leq d} r_{j} \leq 2 e \varepsilon
$$

In other words $\log |P(z)| \geq-d \log (1 / \varepsilon)$ for all $z \in \mathbb{C}$ outside the union of $d$ open discs with radii $\left(r_{j}\right)_{1 \leq j \leq d}$ satisfying the estimate $\sum_{1 \leq j \leq d} r_{j} \leq 2 e \varepsilon$.

From this estimate it is possible to derive the following minimum principle for holomorphic functions (see [26], [35]). If $f$ is a holomorphic function 
on the disc $\{z \in \mathbb{C} ;|z| \leq 2 e R\}$ such that $f(0)=1$. Then for any real number $0<\eta<1$, the following lower bound

$$
\log |f(z)|>-H(\eta) \log M_{f}(2 e R), \text { where } H(\eta):=\log \left(3 e^{3} / 2 \eta\right),
$$

holds for $|z| \leq R$ outside the union of a finite number discs of radii $\left(r_{j}\right)$ with $\sum_{j} r_{j} \leq 2 \eta R$.

There is a more general version of Cartan-Boutroux' lemma which can be stated as follows (see [26]). For any $0<\alpha \leq 2$ there exists a finite covering of $E(P ; \varepsilon)$ by $d$ open discs with radii $\left(r_{j}\right)$ satisfying the estimate

$$
\sum_{j=1}^{d} r_{j}^{\alpha} \leq e(2 \varepsilon)^{\alpha} .
$$

In other words this means that for any $\varepsilon \in] 0,1]$ the lower bound $|P(z)| \geq \varepsilon^{d}$ holds for all $z$ ouside the union of $d$ exceptional discs with radii $\left(r_{j}\right)$ satisfying the estimate $\sum_{j} r_{j}^{\alpha} \leq e(2 \varepsilon)^{\alpha}$.

This is equivalent to the following estimate in terms of the Hausdorff content of dimension $\alpha$

$$
\left.\left.h^{\alpha}(E(P ; \varepsilon)) \leq e(2 \varepsilon)^{\alpha}, \forall \varepsilon \in\right] 0,1\right] .
$$

Let us end this section by recalling the definition of the Hausdorff contents in a more general setting, since it will be used later.

Let $(X, d)$ be a metric space and $p>0$ a real number. Then for a given real number $\delta>0$, by definition, the $\delta$-Hausdorff content of dimension $p$ of a subset $E \subset X$ is defined as follows

$$
h_{\delta}^{p}(E):=\inf \left\{\sum_{j \in \mathbb{N}} r\left(B_{j}\right)^{p} ; E \subset \bigcup_{j \in \mathbb{N}} B_{j},\left(B_{j}\right)_{j \in \mathbb{N}} \in \mathcal{B}_{\delta}(X, d)\right\},
$$

where $\mathcal{B}_{\delta}(X, d)$ is the class of all countable coverings $\left(B_{j}\right)_{j \in \mathbb{N}}$ of the set $E$ by balls of the metric space $(X, d)$ of radii at most $\delta$ and $r\left(B_{j}\right)$ is the radius of the ball $B_{j}$ for each $j \in \mathbb{N}$.

Usually as in the Cartan-Boutroux lemma, we could just take $\delta=+\infty$ to be infinite, which means that in the above definition, we don't ask for an priori bound on the radii $\left(r_{j}\right)$ of the balls $\left(B_{j}\right)$ which cover $E$. The corresponding number is denoted by $h^{p}(E)=h_{\infty}^{p}(E)$ and called the Hausdorff content of dimension $p$ of the set $E$.

Observe that the $p$-dimensional Hausdorff measure of the set $E$ is defined by $H^{p}(E):=\sup _{\delta>0} h_{\delta}^{p}(E)=\lim _{\delta \downarrow 0} h_{\delta}^{p}(E)$.

\section{$3 \quad$ Projective masses and Lelong numbers}

Let us recall some well known definitions and properties concerning Lelong numbers ([25], [15], [19]). 
Let $\Omega$ be a domain in $\mathbb{C}^{n}$ and $P S H(\Omega)$ be the cone of plurisubharmonic functions $u$ on $\Omega$ such that $u \not \equiv-\infty$. Then $P S H(\Omega) \subset L_{l o c}^{1}(\Omega)$ is a closed subset for the $L_{l o c}^{1}$-topology and then it is a complete metric space (see $[19])$.

Let us consider the usual differential operators on $\mathbb{C}^{n}$ defined by $d=\partial+\bar{\partial}$ and define $d^{c}:=(1 / 2 \pi i)(\partial-\bar{\partial})$ so that $d d^{c}=(i / \pi) \partial \bar{\partial}$.

This normalization is choosen so that the following Monge-Ampère equation holds

$$
\left(d d^{c} \log |z|\right)^{n}=\delta(z)
$$

in the sense of currents on $\mathbb{C}^{n}$, where $\delta(z)$ is the Dirac point-mass at the origin.

Now recall that if $V \in P S H(\Omega)$ then $d d^{c} V$ is a closed positive current of bidegree $(1,1)$ on $\Omega$ (see [25]).

For any fixed $a \in \Omega$ and $0<r<<1$ such that $\mathbb{B}(a ; r):=\left\{z \in \mathbb{C}^{n} ; \mid z-\right.$ $a \mid \leq r\} \Subset \Omega$, define the projective mass of the current $d d^{c} V$ on the ball $\mathbb{B}(a, r)$ as follows

$$
\vartheta_{V}(a, r):=\int_{\mathbb{B}(a, r)} d d^{c} V \wedge\left(d d^{c} \log |z-a|\right)^{n-1} .
$$

Then by a well know result of Lelong ([25]), the following formula

$$
\vartheta_{V}(a, r)=\frac{(n-1) !}{\pi^{n-1} r^{2 n-2}} \int_{\mathbb{B}(a, r)} d d^{c} V \wedge \beta_{n-1}=\frac{\mu_{V}(\mathbb{B}(a, r))}{\tau_{2 n-2} r^{2 n-2}}
$$

holds, where $\tau_{2 n-2}$ is the $(2 n-2)$-dimensional volume of the euclidean unit ball in $\mathbb{C}^{n-1}, \beta:=(i / 2) \partial \bar{\partial}|z|^{2}, \beta_{n-1}:=\beta^{n-1} /(n-1) !$ and $\mu_{V}:=(1 / 2 \pi) \Delta V$ is the Riesz measure associated to $V$.

Then the projective mass of the current $d d^{c} V$ at the point $a$ is defined by the following formula

$$
\vartheta_{V}(a):=\lim _{r \rightarrow 0^{+}} \vartheta_{V}(a, r)=\lim _{r \rightarrow 0} \frac{\mu_{V}(\mathbb{B}(a, r))}{\tau_{2 n-2} r^{2 n-2}} .
$$

The positive number $\vartheta_{V}(a)$ is also called the Lelong number of the current $d d^{c} V$ or the Lelong number of the function $V$ at the point $a$.

By a classical result of V. Avanissian (see [3], [20]), the Lelong number can be also expressed by the following formulas

$$
\begin{aligned}
& \vartheta_{V}(a)=\lim _{r \rightarrow 0^{+}} \frac{1}{\log r} \int_{|\xi|=r} V(a+r \xi) d \sigma_{2 n-1}(\xi), \\
& \vartheta_{V}(a)=\lim _{r \rightarrow 0^{+}} \frac{\max _{|z-a|=r} V(z)}{\log r},
\end{aligned}
$$

where $d \sigma_{2 n-1}$ is the normalized area measure on the unit sphere $\partial \mathbb{B}$. 
Observe that when $f$ is a holomorphic function near the point $a$ such that $f \not \equiv 0$ then $\vartheta_{\log |f|}(a)$ is the order of vanishing of $f$ at the point $a$. From the formula (3.5), it follows immediately that $\vartheta_{V}(a)=0$ if $V(a)>-\infty$. This formula shows that the Lelong number $\vartheta_{V}(a)$ can be viewed as the weight of the logarithmic singularity of $V$ at the point $a$.

\section{The minimum principle for logarithmic poten- tials}

Recall that the Lelong class on $\mathbb{C}^{n}$ is defined as follows

$$
\mathcal{L}\left(\mathbb{C}^{n}\right):=\left\{v \in P S H\left(\mathbb{C}^{n}\right) ; v(z) \leq \log ^{+}|z|+O(1), \forall z \in \mathbb{C}^{n}\right\} .
$$

To a function $V \in \mathcal{L}\left(\mathbb{C}^{n}\right)$ there is associated a Robin function as follows (see [27], [37], [6]). For $z \in \mathbb{C}^{n} \backslash\{0\}$, set

$$
\rho_{V}(\zeta):=\limsup _{\lambda \in \mathbb{C}, \lambda \rightarrow \infty}(V(\lambda z)-\log |\lambda z|) .
$$

Since this function is constant on any complex line of $\mathbb{C}^{n}$ passing through the origin, it follows that $\rho_{V}$ is a well defined function on projective space $\mathbb{P}^{n-1}$ which can be viewed as the hyperplane at infinity in $\mathbb{C}^{n}$. Then following Bedford and Taylor ([6]), we introduce the following class.

$$
\mathcal{L}_{\star}\left(\mathbb{C}^{n}\right):=\left\{V \in \mathcal{L}\left(\mathbb{C}^{n}\right) ; \rho_{V} \not \equiv-\infty\right\} .
$$

Let $\omega_{0}$ be the Fubini-Study form on $\mathbb{P}^{n-1}$ normalized by the condition $\int_{\mathbb{P}^{n-1}} \omega_{0}{ }^{n-1}=1$. Then if $V \in \mathcal{L}_{\star}\left(\mathbb{C}^{n}\right)$, the Robin function $\rho_{V}$ is an $\omega_{0}$-plurisubharmonic on $\mathbb{P}^{n-1}$, in the sense that it is upper semi-continuous on $\mathbb{P}^{n-1}$ and satisfies the condition $d d^{c} \rho_{V}+\omega_{0} \geq 0$ in the sense of currents on $\mathbb{P}^{n-1}$.

An interesting fact concerning the class $\mathcal{L}_{\star}\left(\mathbb{C}^{n}\right)$ is the following kind of Riesz representation formula which is well known in one variable but seems not to be known in $\mathbb{C}^{n}$. This formula was obtained earlier with my student Fatima Amghad but never published (see [2]). We will give a proof below since we will use it later.

Lemma 4.1 Any function $V \in \mathcal{L}_{\star}\left(\mathbb{C}^{n}\right)$ admits the following representation formula

$$
V(z)=\int_{\mathbb{C}^{n}} \log |\zeta-z| d d^{c} V \wedge\left(d d^{c} \log |\zeta-z|\right)^{n-1}+\int_{\mathbb{P}^{n-1}} \rho_{V} \omega_{0}^{n-1},
$$

for all $z \in \mathbb{C}^{n}$.

Proof: By translation we may assume that $z=0$ is the origin in $\mathbb{C}^{n}$. By the classical Poisson-Jensen formula, for $0<r<R$, we have

$$
\int_{|\zeta|=1} V(R \zeta) d \sigma_{2 n-1}-\int_{|\zeta|=1} V(r \zeta) d \sigma_{2 n-1}(\zeta)=\int_{r}^{R} \vartheta_{V}(t) \frac{d t}{t}
$$


where $\vartheta_{V}(t):=\vartheta_{V}(0, t)$ is the projective mass of the current $d d^{c} V$ on the ball $\mathbb{B}(0, t)$. From this formula it follows that $V(0)>-\infty$ iff $\int_{0}^{R} \vartheta_{V}(t) \frac{d t}{t}<+\infty$, which implies that $\lim _{t \downarrow 0} \vartheta(t) \log t=0$.

Assume first that $V(0)>-\infty$. Then integration by part gives the following formula

$$
\begin{aligned}
\int_{|\zeta|=1} V(R \zeta) d \sigma_{2 n-1}-\int_{|\zeta|=1} V(r \zeta) d \sigma_{2 n-1}(\zeta)= & \vartheta_{V}(R) \log R-\vartheta_{V}(r) \log r \\
& -\int_{r}^{R} \log t d \vartheta_{V}(t)
\end{aligned}
$$

and then letting $r \downarrow 0$, we get the following formula

$$
\int_{|\zeta|=1} V(R \zeta) d \sigma_{2 n-1}-V(0)=\vartheta_{V}(R) \log R-\int_{r}^{R} \log t d \vartheta_{V}(t) .
$$

Now observe that by approximating $V$ by bounded functions $V_{j}:=\sup \{V,-j\}$ we see from the above formula that $V(0)=-\infty$ iff $\int_{0}^{R} \log t d \vartheta_{V}(t)=-\infty$ and then the formula (4.1) holds also in this case.

Therefore it is enough to prove the formula (4.1) when $V(0)>-\infty$.

In that case, the formula (4.2) yields the following one

$\int_{|\zeta|=1} V(R \zeta) d \sigma_{2 n-1}-\vartheta_{V}(R) \log R=V(0)-\int_{|\zeta|<R} \log |\zeta| d d^{c} V \wedge\left(d d^{c} \log |\zeta|\right)^{n-1}$.

Now since $V \in \mathcal{L}_{\star}\left(\mathbb{C}^{n}\right)$, it follows that

$$
\lim _{R \rightarrow+\infty}\left(\int_{|\zeta|=1} V(R \zeta) d \sigma_{2 n-1}-\log R\right)=\int_{|\zeta|=1} \rho_{V}(\zeta) d \sigma_{2 n-1},
$$

and then

$$
\lim _{R \rightarrow+\infty}(\log R)^{-1} \int_{|\zeta|=1} V(R \zeta) d \sigma_{2 n-1}=1 .
$$

Therefore from Poisson-Jensen's formula it follows immediately that $\lim _{R \rightarrow+\infty} \vartheta_{V}(R)=$ $1, \lim _{R \rightarrow+\infty} \int_{|\zeta|<R} \log |\zeta| d d^{c} V \wedge\left(d d^{c} \log |\zeta|\right)^{n-1}$ is finite and then $\lim _{R \rightarrow+\infty}(1-$ $\left.\vartheta_{V}(R)\right) \log R=0$.

Therefore we deduce the following formula

$$
\int_{|\zeta|=1} \rho_{V}(\zeta) d \sigma_{2 n-1}=V(0)-\int_{\mid \mathbb{C}^{n}} \log |\zeta| d d^{c} V \wedge\left(d d^{c} \log |\zeta|\right)^{n-1}
$$

On the other hand, using Fubini's theorem for the projection $\pi: \mathbb{S}_{2 n-1} \longmapsto$ $\mathbb{P}^{n-1}$, we deduce that

$$
\int_{|\zeta|=1} \rho_{V}(\zeta) d^{c} \log |\zeta| \wedge\left(d d^{c} \log |\zeta|\right)^{n-1}=\int_{\mathbb{P}^{n-1}} \rho_{V} \omega_{0}^{n-1}
$$


Now observe that the normalized area measure $\sigma_{2 n-1}$ on the unit sphere $\mathbb{S}_{2 n-1}$ coincides with the restriction to $\mathbb{S}_{2 n-1}$ of the form $d^{c} \log |\zeta| \wedge\left(d d^{c} \log |\zeta|\right)^{n-1}$, which implies the required formula.

It follows from this formula that the following class of plurisubharmonic functions

$$
\mathcal{L}_{\text {og }}\left(\mathbb{C}^{n}\right):=\left\{V \in \mathcal{L}_{\star}\left(\mathbb{C}^{n}\right) ; \int_{\mathbb{P}^{n-1}} \rho_{V} \omega_{0}^{n-1}=0\right\}
$$

is a natural generalization of the class of classical logarithmic potentials. For this reason we will call this class the class of logarithmic potentials in $\mathbb{C}^{n}$.

Connected with this class there is a natural capacity on $\mathbb{C}^{n}$ which is a generalization of the classical logarithmic capacity. For any subset $E \subset$ $\mathbb{C}^{n}$, recall that the Siciak-Zahariuta's extremal function associated to $E$ is defined by the following formula (see [32], [33], [36]).

$$
V_{E}(z):=\sup \left\{V(z) ; V \in \mathcal{L}\left(\mathbb{C}^{n}\right) ; \sup _{E} V \leq 0\right\}
$$

Then the logarithmic capacity of the set $E$ will be defined by the following formula

$$
C_{\log }(E):=\exp \left(-\int_{\mathbb{P}^{n-1}} \rho_{\left.V_{E}^{*} \omega_{0}^{n-1}\right)}\right.
$$

Observe that if $E$ is pluripolar then $V_{E}^{*} \equiv+\infty$ and then $C_{\log }(E)=0$. On the other hand if $E \Subset \mathbb{C}^{n}$ is non-pluripolar then $V_{E}^{*} \in \mathcal{L}\left(\mathbb{C}^{n}\right)$ and $\rho_{V_{E}^{*}}$ is bounded on $\mathbb{P}^{n-1}$, which proves that $C_{\log }(E)>0$.

Moreover using a result concerning the convergence of Robin's functions from [6], it's possible to prove that this logarithmic capacity is a Choquet capacity on $\mathbb{C}^{n}$.

Observe that this capacity is related to the class of logarithmic potentials by the following formula

$$
\log C_{\log }(E)=\inf \left\{\sup _{E}^{\star} V ; V \in \mathcal{L}_{o g}\left(\mathbb{C}^{n}\right)\right\},
$$

where $\sup _{E}{ }^{\star} V:=\inf \left\{\sup _{E \backslash A} V ; A \subset E\right.$ is pluripolar $\}$ is the quasi-essential upper bound of $V$ on the set $E$. Indeed, it is easy to see from the definition of $V_{E}^{\star}$ and results of [5] that $\sup _{E}^{\star} V_{E}^{\star}=0$ and then

$$
V_{E}^{\star}(z)=\sup \left\{V(z) ; V \in \mathcal{L}\left(\mathbb{C}^{n}\right) ; \sup _{E}^{\star} V=0\right\}, \quad z \in \mathbb{C}^{n} .
$$

Therefore we easily get the following formula

$$
\begin{aligned}
-\log C_{\log }(E) & =\sup \left\{\int_{\mathbb{P}^{n-1}} \rho_{V} \omega_{0}^{n-1} ; V \in \mathcal{L}\left(\mathbb{C}^{n}\right), \sup _{E}^{\star} V=0\right\} \\
& =\sup \left\{\int_{\mathbb{P}^{n-1}} \rho_{V} \omega_{0}^{n-1}-\sup _{E}^{\star} V ; V \in \mathcal{L}\left(\mathbb{C}^{n}\right)\right\},
\end{aligned}
$$


where the supremum is attained for $V=V_{E}^{\star}$.

Now from the formula (4.4), it follows that

$$
\log C_{\log }(E)=\inf \left\{\sup _{E}^{\star} V-\int_{\mathbb{P}^{n-1}} \rho_{V} \omega_{0}^{n-1} ; V \in \mathcal{L}\left(\mathbb{C}^{n}\right)\right\},
$$

which proves the formula (4.3).

Recall that there is another constant which was classically called the logarithmic capacity and defined for a compact subset $K \subset \mathbb{C}^{n}$ by the following formula ([33], [27], [22], [37])

$$
\tau(K):=\exp \left(-\limsup _{|z| \rightarrow+\infty}\left(V_{K}^{\star}(z)-\log |z|\right)=\exp \left(-\sup _{|z|=1} \rho_{V_{K}^{\star}}\right) .\right.
$$

From a well known inequality (see [1], [14], [33]), it follows easily that there exists a constant $\kappa_{n}>0$ such that

$$
\tau(K) \leq C_{\log }(K) \leq \kappa_{n} \cdot \tau(K)
$$

for any compact subset $K \subset \mathbb{C}^{n}$.

We want to prove the following generalization of the Cartan-Boutroux lemma for the class $\mathcal{L}_{o g}\left(\mathbb{C}^{n}\right)$ of logarithmic potentials.

Theorem 4.2 For any real number $0<\eta<5$ and any function $V \in$ $\mathcal{L}_{\text {og }}\left(\mathbb{C}^{n}\right)$, the following lower bound

$$
V(z) \geq-\log (5 e / \eta)
$$

holds for all $z \in \mathbb{C}^{n}$, outside the union of a countable family of euclidean balls $\left(\mathbb{B}\left(z_{j}, r_{j}\right)\right)$ of radii $\left(r_{j}\right)$ less than $\eta$, satisfying the following condition

$$
\sum_{\mathbb{B}\left(z_{j}, r_{j}\right) \cap \mathbb{B}_{R} \neq \emptyset} r_{j}^{2 n-2+\alpha}<5^{2 n-2}(R+\eta)^{2 n-2} \eta^{\alpha} / \alpha, \forall R>0 .
$$

In particular, the exceptionnal set $E_{\eta} \subset \mathbb{C}^{n}$ where the lower estimate (4.5) is not satisfied is a Borel set for which the following estimate

$$
h_{\eta}^{2 n-2+\alpha}\left(E_{\eta} \cap \mathbb{B}_{R}\right)<5^{2 n-2}(R+\eta)^{2 n-2} \eta^{\alpha} / \alpha, \forall R>0,
$$

holds.

Proof: Let $V \in \mathcal{L}_{o g}\left(\mathbb{C}^{n}\right)$. First observe that considering the Stieltjes' integral with respect to the increasing function $g: t \longmapsto \vartheta_{V}(z, t)$, we can write

$$
V(z)=\int_{0}^{+\infty} \log t d g(t) \geq \int_{0}^{1} \log t d g(t), \forall z \in \mathbb{C}^{n} .
$$

Now fix the real numbers $0<\alpha \leq 2$ and $0<\varepsilon<1 / 5$ and let $A>0$ be a real number to be specified later in terms of $\varepsilon$ and $\alpha$. Then denote by $G=G_{\varepsilon, \alpha}$ 
the subset of "good" points $z \in \mathbb{C}^{n}$ for which we have the following bound on the projective mass

$$
\vartheta_{V}(z, t) \leq A t^{\alpha}, \forall 0<t \leq \varepsilon .
$$

Observe that this implies in particular that $\lim _{t \rightarrow 0} \vartheta_{V}(z, t) \log t=0$ for $z \in G$, which implies that $V(z)>-\infty$ for $z \in G$ and thus the set $G$ doesn't meet the polar set of $V$.

Now fix a point $z \in G$. Then $V(z)>-\infty$ and integration by parts in the Stieltjes' integral implies immediately that

$$
\begin{aligned}
V(z) & \geq-\int_{0}^{1} \vartheta_{V}(z, t) \frac{d t}{t} \\
& \geq-\int_{0}^{\varepsilon} \vartheta_{V}(z, t) \frac{d t}{t}-\int_{\varepsilon}^{1} \vartheta_{V}(z, t) \frac{d t}{t} \\
& \geq-\frac{A}{\alpha} \varepsilon^{\alpha}-\int_{\varepsilon}^{1} \vartheta_{V}(z, t) \frac{d t}{t} .
\end{aligned}
$$

Since $V \in \mathcal{L}\left(\mathbb{C}^{n}\right)$ and due to our normalisation of the complex MongeAmpère operator, we know that

$$
\vartheta_{V}(z, t) \leq \int_{\mathbb{C}^{n}} d d^{c} V \wedge\left(d d^{c} \log |z-\zeta|\right)^{n-1} \leq 1, \forall z \in \mathbb{C}^{n}, \forall t>0 .
$$

Therefore we derive the following estimate

$$
V(z) \geq-\frac{A}{\alpha} \varepsilon^{\alpha}-\log (1 / \varepsilon), \forall z \in G .
$$

Choosing $A:=\alpha \varepsilon^{-\alpha}$, we obtain the following lower bound

$$
V(z) \geq-\log (e / \varepsilon), \forall z \in G .
$$

Therefore we obtain the required inequality if we can estimate properly the size of the exceptionnel set $E:=\mathbb{C}^{n} \backslash G$.

From the definition of the set $E$, it follows that for any $z \in E$ there exists a real number $0<t_{z}<\varepsilon$ such that

$$
\vartheta_{V}\left(z, t_{z}\right)>A t_{z}^{\alpha}
$$

On the other hand, an easy computation shows that

$$
r^{2-2 n} \mu_{V}(\mathbb{B}(z, r))=\vartheta_{V}(z, r) \leq 1, \forall z \in \mathbb{C}^{n}, \forall r>0 .
$$

Therefore we get

$$
\mu_{V}\left(\mathbb{B}\left(z, t_{z}\right)\right) \geq t_{z}^{2 n-2} \vartheta_{V}\left(z, t_{z}\right)>A t_{z}^{2 n-2+\alpha}, \forall z \in E .
$$


We want to exclude such balls. Since $\left(\mathbb{B}\left(z, t_{z}\right)\right)_{z \in E}$ is a covering of the exceptionnal set $E$ by open euclidean balls, by a Vitalli type 5 -covering lemma (see [29]), there exists a countable subfamily of mutually disjoint balls $\left(\mathbb{B}\left(z_{j}, t_{j}\right)\right)_{j \in \mathbb{N}}$ such that the corresponding 5 -family of balls $\left(\mathbb{B}\left(z_{j}, 5 t_{j}\right)\right)_{j \in \mathbb{N}}$ covers $E$.

Now fix $R>0$ and consider the familly $\left(\mathbb{B}\left(z_{j}, 5 t_{j}\right)\right)_{j \in J_{R}}$ of those balls which intersect the ball $\mathbb{B}_{R}$. Then we obtain the following estimate

$$
\left.A \sum_{j \in J_{R}}\left(5 t_{j}\right)^{2 n-2+\alpha}<5^{2 n-2+\alpha} \sum_{j \in J_{R}} t_{j}^{2 n-2} \vartheta_{V}\left(z_{j}, t_{j}\right)\right) \leq 5^{2 n-2+\alpha} \sum_{j \in J_{R}} \mu_{V}\left(\mathbb{B}\left(z_{j}, t_{j}\right)\right) .
$$

Observing that

$$
\sum_{j \in J_{R}} \mu_{V}\left(\mathbb{B}\left(z_{j}, t_{j}\right)\right)=\mu_{V}\left(\bigcup_{j \in J_{R}} \mathbb{B}\left(z_{j}, t_{j}\right) \leq \mu_{V}(\mathbb{B}(0, R+\varepsilon)) \leq(R+\varepsilon)^{2 n-2},\right.
$$

we conclude that

$$
\sum_{j \in J_{R}}\left(5 t_{j}\right)^{2 n-2+\alpha}<(5 R+1)^{2 n-2} 5^{\alpha} / A=(5 R+1)^{2 n-2}(5 \varepsilon)^{\alpha} / \alpha .
$$

Taking $r_{j}:=5 t_{j}$ and $\varepsilon:=\eta / 5$, we obtain the theorem since the family of balls $\left(\mathbb{B}\left(z_{j}, r_{j}\right)\right)_{j \in J_{R}}$ covers $E \cap \mathbb{B}_{R}$

Observe that the above result yields immediately a precise estimate on the $2 n-2+\alpha-$ Hausdorff contents of the plurisubharmonic lemniscates associated to functions in the class $\mathcal{L}_{\star}\left(\mathbb{C}^{n}\right)$.

Corollary 4.3 Let $V \in \mathcal{L}_{\star}\left(\mathbb{C}^{n}\right)$ and $0<\varepsilon<1 /$. Then for any $\alpha \in$ ]0,2], the $2 n-2+\alpha$-Hausdorff content of the associated plurisubharmonic lemniscate $E(V, \varepsilon):=\left\{z \in \mathbb{C}^{n} ; V(z) \leq \log \varepsilon\right\}$ satifies the following estimates

$$
h_{\eta}^{2 n-2+\alpha}\left(E_{\varepsilon} \cap \mathbb{B}_{R}\right)<5^{2 n-2}(R+5)^{2 n-2}(5 e \varepsilon)^{\alpha} / \alpha, \forall R>0,
$$

holds.

From the theorem it's also possible to deduce the following comparison inequality between certain relative Hausdorff contents and the logarithmic capacity defined above.

Corollary 4.4 For any real number $0<\alpha \leq 2$ and any subset $K \subset \mathbb{B}$, we have

$$
h^{2 n-2+\alpha}(K) \leq \frac{c_{n}}{\alpha}\left(5 e C_{\log }(K)\right)^{\alpha},
$$

where $c_{n}=5^{2 n-2}(1+1 / e)^{2 n-2}$.

Proof: Recall from the formula (4.3) that

$$
\log C_{\log }(K)=\inf \left\{\sup _{K}^{\star} V ; V \in \mathcal{L}^{+}\left(C^{n}\right) ; \int_{\mathbb{P}^{n-1}} \rho_{V} \omega_{0}^{n-1}=0\right\},
$$


where $\sup _{K}^{\star} V$ is the quasi-essential upper bound of $V$ on $K$.

Assume first that $K \subset \mathbb{B}_{r}$ with $r:=1 / e$ so that $C_{\log }(K)<1 / e$. Then let $c$ be an arbitrary real number such that $C_{\log }(K)<c<1 / e$. Then there exists a function $V \in \mathcal{L}_{o g}\left(\mathbb{C}^{n}\right)$ and a pluripolar subset $A \subset E$ such that $\sup _{K \backslash A} V<c$ so that $K \backslash A \subset K_{c}:=\left\{z \in \mathbb{B}_{r} ; V(z)<\log c\right\}$. By the minimum principle for the class $\mathcal{L}_{o g}\left(\mathbb{C}^{n}\right)$, with $\eta=5 e c<5$, we have $V(z) \geq-\log (5 e / \eta)=\log c$ for $z \in \mathbb{B} \backslash E$, where $E \subset \mathbb{B}$ is a Borel set satisfying $h^{2 n-2+\alpha}(E) \leq 5^{2 n-2}(r+\eta / 5)^{2 n-2} \eta^{\alpha} / \alpha=5^{2 n-2}(r+1)^{2 n-2}(5 e c)^{\alpha} /$ $\alpha$. Since by definition $K \backslash A \subset K_{c} \subset E$, we deduce that $h^{2 n-2+\alpha}(K \backslash A) \leq$ $(5 r+1)^{2 n-2}(5 e c)^{\alpha} / \alpha$.

Since $c>C_{\log }(K)$ is arbitrary, we obtain the inequality

$$
h^{2 n-2+\alpha}(K \backslash A) \leq(5 r+1)^{2 n-2}\left(5 e C_{\log }(K)\right)^{\alpha} / \alpha .
$$

Since $A \in \mathbb{C}^{n}$ is pluripolar, it is polar in $\mathbb{R}^{2 n}$ and then from a well known result in classical potential theory (see [23]), it follows that $h^{2 n-2+\alpha}(A)=0$ and then $h^{2 n-2+\alpha}(K)=h^{2 n-2+\alpha}(K \backslash A)$, which proves the required estimate in the case when $K \subset B_{r}$.

Now if $K \subset \mathbb{B}$ is any subset, it is enough to apply the last inequality to the set $r \cdot K \subset r \mathbb{B}$ to obtain the required inequality.

Remarks: 1) Observe that if $n=1$ and $\alpha=1$, our estimate 4.7) reduces precisely to that given in [26]. This extends the Cartan-Boutroux lemma except that the constant in our estimate is $5 e$ instead of $2 e$.

2 ) When $n \geq 2$ our estimate shows how the relative Hausdorff content of the exceptionnal set with respect to the ball $\mathbb{B}_{R}$ is asymptotically small when $R \rightarrow+\infty$.

3) Observe that for $\alpha=2$ similar estimates in terms of the relative logarithmic capacity was obtained in [8].

\section{A lower bound for plurisubharmonic functions}

Let $\mathbb{B}$ be the euclidean unit open ball in $\mathbb{C}^{n}$. For each $z \in \mathbb{B}$, we denote by $\Phi_{z}$ the involutive automorphism of the unit ball $\mathbb{B}$ which takes the point $z \in \mathbb{B}$ to the origin. Then the pluricomplex Green function $G_{z}(\zeta):=G(\zeta, z)$ of the unit ball $\mathbb{B}$ with a logarithmic pole at the point $z \in \mathbb{B}$ is given by the formula

$$
G_{z}(\zeta):=\log \left|\Phi_{z}(\zeta)\right|, \quad(z, \zeta) \in \mathbb{B} \times \mathbb{B}
$$

It is easy to see that the following fundamental Monge-Ampère equation

$$
\left(d d^{c} G_{z}\right)^{n}=\delta_{z}
$$

holds in the sense of currents on $\mathbb{B}$, where $\delta_{z}$ is the unit Dirac mass at the point $z$. 
It is well known that the formula $d_{\mathbb{B}}(z, \zeta):=\left|\Phi_{z}(\zeta)\right|$ defines a distance on the unit ball $\mathbb{B}$ which is related to the Bergman distance $\rho_{\mathbb{B}}$ by the following formula

$$
d_{\mathbb{B}}(z, \zeta)=\tanh \frac{\rho_{\mathbb{B}}(z, \zeta)}{\sqrt{n+1}} .
$$

Now consider the corresponding ball of center $z \in \mathbb{B}$ and radius $r \in] 0,1[$ defined by

$$
\omega_{z}(r):=\left\{\zeta \in \mathbb{B} ;\left|\Phi_{z}(\zeta)\right|<r\right\}
$$

and define the following "invariant projective mass" function

$$
\theta_{V}(z, r):=\int_{\omega_{z}(r)} d d^{c} V \wedge\left(d d^{c} G_{z}\right)^{n-1}
$$

for $z \in \mathbb{B}$ and $0<r<1$ and observe that

$$
\theta_{V}(z, r)=\int_{\mathbb{B}_{r}} d d^{c} V \circ \Phi_{z} \wedge\left(d d^{c} \log |\zeta|\right)^{n-1}=\vartheta_{V \circ \Phi_{z}}(0, r),
$$

and then $\lim _{r \rightarrow 0} \theta_{V}(z, r)=\vartheta_{V \text { o } \Phi_{z}}(0)=\vartheta_{V}(z)$ for any $z \in \mathbb{B}$ since $\Phi_{z}$ is an automorphism taking the origin to the point $z$ (see [15], [19]).

First we prove the following lemma which is similar to a result of $\mathrm{H}$. Milloux concerning a lower estimate for monic polynomials with all zeros in the unit disc, the exceptional set being estimated in terms of non euclidean distance on the unit disc (see [30]).

Lemma 5.1 Let $V$ be a plurisubharmonic function on the euclidean open unit ball $\mathbb{B} \subset \mathbb{C}^{n}$ with bounded Riesz mass $\mu_{V}(\mathbb{B}):=(1 / 2 \pi) \int_{\mathbb{B}} \Delta V<+\infty$. Let us define the following pluricomplex Green potential

$$
\mathcal{G}_{V}(z):=\int_{\mathbb{B}} G_{z} d d^{c} V \wedge\left(d d^{c} G_{z}\right)^{n-1}, z \in \mathbb{B} .
$$

Then there exists a constant $c_{n}>0$ such that for any real numbers $0<s<1$ and $0<\eta<\min \{3 s, 1\}$, the following lower bound

$$
\mathcal{G}_{V}(z) \geq-\theta_{V}(z, s) \log (3 / \eta)-c_{n} \mu_{V}(\mathbb{B}) \log (e / s),
$$

holds for all $z \in \mathbb{B}$, outside the union of a countable family of pseudo-balls $\left(\omega_{z_{j}}\left(r_{j}\right)\right)$ of radii $\left(r_{j}\right)$ less than $\eta$ and satisfying the condition $\sum_{j} r_{j}^{2 n-2+\alpha}<$ $9^{n-1} \eta^{\alpha} / \alpha$.

In particular, the exceptionnal set $E \subset \mathbb{B}$ where the lower bound (5.9) does not hold is a Borel set whose invariant $\eta-H a u s d o r f f$ content of dimension $2 n-2+\alpha$ satisfies the estimate $\tilde{h}_{\eta}^{2 n-2+\alpha}(E)<9^{n-1} \eta^{\alpha} / \alpha$. 
Proof: To estimate the function $\mathcal{G}_{V}$ given by the formula (5.5), observe that by considering the Stieltjes' integral with respect to the increasing function $g: t \longmapsto \theta_{V}(z, t)$, we can write the formula (5.5) as follows

$$
\mathcal{G}_{V}(z)=\int_{0}^{1} \log t d g(t), \forall z \in \mathbb{B} .
$$

Now fix the real numbers $0<\alpha \leq 2$ and $0<\varepsilon<\min \{s, 1 / 3\}$ and let $A>0$ be a real number to be specified later in terms of $\varepsilon$ and $\alpha$. Then denote by $U=U_{\varepsilon, \alpha}$ the subset of "good" points $z \in \mathbb{B}$ for which we have the following bound on the invariant projective mass

$$
\theta_{V}(z, t) \leq A t^{\alpha}, \forall 0<t \leq \varepsilon .
$$

Observe that this implies in particular that $\vartheta_{V}(z)=0$ for $z \in U$, which shows that the set $U$ doesn't contain the logarithmic singularities of $V$.

Now fix a point $z \in U$. Then integration by parts in the Stieltjes' integral implies immediately that

$$
\begin{aligned}
\mathcal{G}_{V}(z) & =-\int_{0}^{1} \theta_{V}(z, t) \frac{d t}{t} \\
& =-\int_{0}^{\varepsilon} \theta_{V}(z, t) \frac{d t}{t}-\int_{\varepsilon}^{1} \theta_{V}(z, t) \frac{d t}{t} \\
& \geq-\frac{A}{\alpha} \varepsilon^{\alpha}-\int_{\varepsilon}^{1} \theta_{V}(z, t) \frac{d t}{t} .
\end{aligned}
$$

On the other hand, we can write

$$
\int_{\varepsilon}^{1} \theta_{V}(z, t) \frac{d t}{t}=\int_{\varepsilon}^{s} \theta_{V}(z, t) \frac{d t}{t}+\int_{s}^{1} \theta_{V}(z, t) \frac{d t}{t} .
$$

Now observe that

$$
\int_{\varepsilon}^{s} \theta_{V}(z, t) \frac{d t}{t} \leq \theta_{V}(z, s) \log (s / \varepsilon)
$$

and

$$
\int_{s}^{1} \theta_{V}(z, t) \frac{d t}{t} \leq \theta_{V}(z, 1) \log (1 / s) .
$$

Therefore we conclude that

$$
\mathcal{G}_{V}(z) \geq-\frac{A}{\alpha} \varepsilon^{\alpha}-\theta_{V}(z, s) \log (s / \varepsilon)-\theta_{V}(z, 1) \log (1 / s), \forall z \in U .
$$

Choosing $A:=\alpha c_{n} \mu_{V}(\mathbb{B}) \varepsilon^{-\alpha}$, we obtain the following lower bound

$$
\mathcal{G}_{V}(z) \geq-c_{n} \mu_{V}(\mathbb{B})-\theta_{V}(z, s) \log (s / \varepsilon)-c_{n} \mu_{V}(\mathbb{B}) \log (1 / s), \forall z \in U .
$$


Therefore we obtain the required inequality if we can estimate properly the size of the exceptionnel set $E:=\mathbb{B} \backslash U$.

From the definition of the set $E$, it follows that for any $z \in E$ there exists a real number $0<t_{z}<\varepsilon$ such that

$$
\theta_{V}\left(z, t_{z}\right)>A t_{z}^{\alpha}
$$

On the other hand, an easy computation shows that there exists a constant $c_{n}>0$ such that

$$
\left.\left.\theta_{V}(z, r) \leq c_{n} r^{2-2 n} \mu_{V}\left(\omega_{z}(r)\right)\right), \forall z \in \mathbb{B}, \forall r \in\right] 0,1 / 3[.
$$

Therefore we get

$$
c_{n} \mu_{V}\left(\omega_{z}\left(t_{z}\right)\right) \geq t_{z}^{2 n-2} \theta_{V}\left(z, r_{z}\right)>A t_{z}^{2 n-2+\alpha}, \forall z \in E .
$$

Since $\left(\omega_{z}\left(t_{z}\right)\right)_{z \in E}$ is a covering of the exceptionnal set $E$ by invariant pseudoballs, by an elementary Vitalli type 3 -covering lemma for the ball with the hyperbolic distance (see [31], [34]), there exists a countable subfamily of mutually disjoint pseudo-balls $\left(\omega_{z_{j}}\left(t_{j}\right)\right)$ such that the corresponding 3 -family of pseudo-balls $\left(\omega_{z_{j}}\left(3 t_{j}\right)\right)$ covers $E$. Moreover we obtain the following estimate

$\left.A \sum_{j}\left(3 t_{j}\right)^{2 n-2+\alpha}<3^{2 n-2+\alpha} \sum_{j} t_{j}^{2 n-2} \theta_{V}\left(z_{j}, t_{j}\right)\right) \leq 3^{2 n-2+\alpha} c_{n} \sum_{j} \mu_{V}\left(\omega_{z_{j}}\left(t_{j}\right)\right)$.

Observing that $\sum_{j} \mu_{V}\left(\omega_{z_{j}}\left(t_{j}\right)\right)=\mu_{V}\left(\bigcup \omega_{z_{j}}\left(t_{j}\right)\right) \leq \mu_{V}(\mathbb{B})$, we conclude that

$$
\sum_{j}\left(3 t_{j}\right)^{2 n-2+\alpha}<9^{n-1} 3^{\alpha} c_{n} \mu_{V}(\mathbb{B}) / A=9^{n-1}(3 \varepsilon)^{\alpha} / \alpha .
$$

Taking $r_{j}:=3 t_{j}$ and $\varepsilon:=\eta / 3$, we obtain the theorem since the family of pseudo-balls $\left(\omega_{z_{j}}\left(r_{j}\right)\right)$ covers $E$

As a consequence of the last lemma we obtain a minimum principle for the Cegrell's class $\mathcal{F}(\mathbb{B})([12])$ on the unit ball in terms of the pseudo-distance on the unit ball.

Proposition 5.2 Let Then there exists a constant $c_{n}>0$ such that for any real numbers $0<\eta<1$ and any function $\varphi \in \mathcal{F}(\mathbb{B})$ with $\int_{\mathbb{B}}\left(d d^{c} \varphi\right)^{n} \leq 1$, the following lower bound

$$
\varphi(z) \geq-\log \left(c_{n} / \eta\right)
$$

holds for all $z \in \mathbb{B}$, outside the union of a countable family of pseudo-balls $\left(\omega_{z_{j}}\left(r_{j}\right)\right)$ of radii $\left(r_{j}\right)$ less than $\eta$ and satisfying the condition

$$
\sum_{j} r_{j}^{2 n-2+\alpha}<9^{n-1} \eta^{\alpha} / \alpha
$$


In particular, the exceptionnal set $E \subset \mathbb{B}$ where the lower bound (5.9) does not hold is a Borel set whose invariant $\eta$-Hausdorff content of dimension $2 n-2+\alpha$ satisfies the estimate

$$
\tilde{h}_{\eta}^{2 n-2+\alpha}(E)<9^{n-1} \eta^{\alpha} / \alpha .
$$

Proof: From the definition of the class $\mathcal{F}(\mathbb{B})$ (see [12]), it follows that there exists a decreasing sequence of plurisubharmonic functions on $\mathbb{B}$ with boundary values 0 which converges to $\varphi$ and satisfies the condition $\sup _{j} \int_{\mathbb{B}}\left(d d^{c} \varphi_{j}\right)^{n}<$ $+\infty$. Applying the Poisson-Jensen formula to every $\varphi_{j}$ and taking the limit we obtain the formula $\varphi(z)=\mathcal{G}_{\varphi}(z)$ for $z \in \mathbb{B}$.

It's well known that functions from the class $\mathcal{F}(\mathbb{B})$ have bounded Riesz mass (see [12]) and then we can apply the last lemma to conclude that the estimate (5.9) holds for $V=\varphi$ and $s=1$. Now oberve that $\mu_{\varphi}(\mathbb{B}) \leq 1$ and $\theta_{\varphi}(z, 1) \leq 1$ if $\varphi \in \mathcal{F}(\mathbb{B})$ and $\int_{\mathbb{B}}\left(d d^{c} \varphi\right)^{n} \leq 1$ (see $[\mathrm{Ce}]$ ), which implies the required estimates.

Now let us state the main result of this section. For a fixed $0<\rho<1$, define the following constant

$$
\kappa_{n}(\rho):=\frac{(1+\rho)^{n}}{(1-\rho)^{n}}
$$

Theorem 5.3 Let $0<\rho<1$ be a real number. Then there exists a positive constant $c_{n}(\rho)>1$ such that for any real numbers $0<s<1,0<\eta<$ $\min \{s, 1 / 3\}$ and any function $V$ plurisubharmonic on a neighbourhood of the closed euclidean unit ball $\overline{\mathbb{B}} \subset \mathbb{C}^{n}$ such that $V \leq 0$ on $\mathbb{B}$, the following lower bound

$$
V(z) \geq \kappa_{n}(\rho) \int_{\partial \mathbb{B}} V d \sigma_{2 n-1}-\theta_{V}(z, s) \log (3 / \eta)-c_{n}(\rho) \mu_{V}(\mathbb{B}) \log (e / s)
$$

holds for all $z \in \mathbb{B}_{\rho}$, outside the union of a countable family of pseudo-balls $\left(\omega_{z_{j}}\left(r_{j}\right)\right)$ of radii $\left(r_{j}\right)$ not exceding $\eta$ and satisfying the condition $\sum_{j} r_{j}^{2 n-2+\alpha}<$ $9^{n-1} \eta^{\alpha} / \alpha$.

In particular, the exceptionnal set $E \subset \mathbb{B}_{\rho}$ where the lower bound (5.10) does not hold is a Borel set whose Hausdorff content satisfies the estimate $h_{\eta}^{2 n-2+\alpha}(E)<9^{n-1} \eta^{\alpha} / \alpha$.

Proof: By the Jensen-Poisson-Szegö formula, we get the following representation formula

$$
V(z)=\int_{\partial \mathbb{B}} V d^{c} G_{z} \wedge\left(d d^{c} G_{z}\right)^{n-1}+\int_{\mathbb{B}} G_{z} d d^{c} V \wedge\left(d d^{c} G_{z}\right)^{n-1},
$$

for $z \in \mathbb{B}$ (see [31], [34]). Recall that this formula follows easily from the fundamental equation (5.2) and the fact that $G_{z}$ has boundary values 0 . 
Now let us write $V=\mathcal{P}_{V}+\mathcal{G}_{V}$ on $\mathbb{B}$, where

$$
\mathcal{P}_{V}(z):=\int_{\partial \mathbb{B}} V d^{c} G_{z} \wedge\left(d d^{c} G_{z}\right)^{n-1}, z \in \mathbb{B},
$$

and

$$
\mathcal{G}_{V}(z):=\int_{\mathbb{B}} G_{z} d d^{c} V \wedge\left(d d^{c} G_{z}\right)^{n-1}, z \in \mathbb{B} .
$$

It is well known that

$$
d^{c} G_{z} \wedge\left(d d^{c} G_{z}\right)^{n-1}=\mathcal{P}(z, .) d \sigma_{2 n-1},
$$

where

$$
\mathcal{P}(z, \zeta):=\frac{\left(1-|z|^{2}\right)^{n}}{|1-z \cdot \bar{\zeta}|^{2 n}},(z, \zeta) \in \mathbb{B} \times \partial \mathbb{B},
$$

is the Poisson-Szegö kernel of the open unit ball $\mathbb{B}$ and $d \sigma_{2 n-1}$ is the normalized area measure on the unit sphere $\mathbb{S}_{2 n-1}=\partial \mathbb{B}$.

Therefore, since $V \leq 0$ on $\mathbb{B}$, it follows that the function $\mathcal{P}_{V}$ satisfies the following lower estimate

$$
\mathcal{P}_{V}(z) \geq \kappa_{n}(\rho) \int_{\partial \mathbb{B}} V(\zeta) d \sigma_{2 n-1}(\zeta), \text { for }|z| \leq \rho .
$$

Then the estimate of the theorem follows from the lemma

\section{A minimum principle for plurisubharmonic func- tions}

Here we will give a general version of the minimum principle for compact classes of plurisubharmonic functions and derive a kind of three-circle minimum principle for arbitrary plurisubharmonic functions.

Theorem 6.1 Let $\Omega \subset \mathbb{C}^{n}$ be an open set, $K \subset \Omega$ a compact set and let $\mathcal{U} \subset$ $P S H(\Omega)$ be a compact class. Then $\left.\vartheta:=\sup \left\{\vartheta_{u}(z) ; u \in \mathcal{U}, z \in K\right\}<+\infty\right\}$ and for any $\nu>\vartheta$ there exists constants $C=C(K, \nu)>0$ and $0<\eta_{0}<1$ such that for any real number $0<\eta \leq \eta_{0}$, any real number $0<\alpha \leq 2$ and any function $u \in \mathcal{U}$ the following lower bound

$$
u(z) \geq-\nu \log (C / \eta),
$$

holds for any $z \in K$ outside the union of a countable family of balls of radii $\left(r_{j}\right)$ less than $\eta$ which satisfy the following condition

$$
\sum_{j} r_{j}^{2 n-2+\alpha}<N 9^{n-1} \eta^{\alpha} / \alpha
$$

where $N$ is an integer depending only on $(K, \Omega)$. 
Proof: By upper semi-continuity of Lelong numbers, there exits a real number $0<s<1$ small enough and an open neighbourhood $\omega \Subset \Omega$ of $K$ such that $\theta_{u}(z, s)<\nu$ for any $z \in \omega$ and any $u \in \mathcal{U}$ (see [39]). Now take a finite number of concentric euclidean balls $B_{i}^{\prime} \Subset \mathbb{B}_{i} \Subset \omega(1 \leq i \leq N)$ such that the balls $\left(\mathbb{B}_{i}^{\prime}\right)_{1 \leq i \leq N}$ cover $K$. By Theorem 5.2, for each $i$ there exits constants $\kappa_{i}>0$ and $c_{i}>0$ such that the following lower bound

$$
V(z) \geq \kappa_{i} \int_{\partial \mathbb{B}_{i}} V d \sigma_{2 n-1}-\theta_{V}(z, s) \log (3 / \eta)-c_{i} \mu_{V}\left(\mathbb{B}_{i}\right) \log (e / s),
$$

holds for all $z \in \mathbb{B}_{i}^{\prime} \backslash E_{i}$, where $E_{i} \subset \mathbb{B}^{\prime} i$ is a Borel set with $h_{\eta}^{2 n-2+\alpha}\left(E_{i}\right)<$ $9^{n-1} \eta^{\alpha} / \alpha$. By compactness of $\mathcal{U}$ it follows that $\int_{\partial \mathbb{B}_{i}} V d \sigma_{2 n-1}$ and $\mu_{V}\left(\mathbb{B}_{i}\right)$ are uniformly bounded for $V \in \mathcal{U}$. Therefore taking $E=\bigcup_{1 \leq i \leq N} E_{i}$, we obtain for a suitable constant $C>0$ the following lower bound

$$
V(z) \geq-\theta_{V}(z, s) \log (3 / \eta)-C \geq \nu \log (3 / \eta)-C,
$$

for any $z \in K \backslash E$. Since $h_{\eta}^{2 n-2+\alpha}(E) \leq \sum_{i} h_{\eta}^{2 n-2+\alpha}\left(E_{i}\right)<N 9^{n-1} \eta^{\alpha} / \alpha$, we obtain the estimate of the theorem with an appropriate constant.

Recall that the class $\mathcal{U}_{\mathbb{B}}:=\left\{v \in \mathcal{L}\left(\mathbb{C}^{n}\right) ; \sup _{\overline{\mathbb{B}}} v=0\right\}$ is a compact class of plurisubharmonic functions. Applying the same proof as in the last theorem we obtain the following result which will be called the minimum principle for the Lelong class.

Theorem 6.2 For any $\nu>1$ there exists constants $C>0$ and $0<\eta_{0}<1$ such that for any real number $0<\eta \leq \eta_{0}$, any real number $0<\alpha \leq 2$, any real number $R \geq 1$ and any $V \in \mathcal{L}\left(\mathbb{C}^{n}\right)$, the following lower bound

$$
V(z) \geq \max _{B_{R}} V-\nu \log (C / \eta)
$$

holds for any $z \in \mathbb{B}_{R}$ outside the union of a countable family of euclidean balls of radii $\left(r_{j}\right)$ less than $\eta R$ which satisfy the following condition

$$
\sum_{j} r_{j}^{2 n-2+\alpha}<9^{n-1} R^{2 n-2+\alpha} \eta^{\alpha} / \alpha .
$$

Proof: It is enough to prove the estimate for $R=1$. Then applying the same method as in the proof of the previous theorem to the compact class $\mathcal{U}_{\mathbb{B}}$ and observing that for any $v \in \mathcal{L}\left(\mathbb{C}^{n}\right), u:=v-\sup _{\overline{\mathbb{B}}} v \in \mathcal{U}_{\mathbb{B}}$ we obtain the required lower bounds since in this cas $N=1$.

We want to apply the minimum principle for compact classes to prove the following general result which extends the classical minimum principle stated in section 2 . 
To motivate our result, let us recall that the maximum principle for plurisubharmonic functions can be used to prove the classical Hadamard three-circle inequality which can be stated as follows. Let $V$ be a plurisubharmonic function on the euclidean ball $\mathbb{B}_{R}$ of radius $R>0$ and let $\sigma, \tau \in$ ] 0,1 [ be real numbers with $0<\sigma \leq \tau<1$. Then the following upper bound

$$
V(z) \leq \sup _{\mathbb{B}_{\sigma R}} V+\rho(\sigma, \tau)\left(\sup _{\mathbb{B}_{R}} V-\sup _{\mathbb{B}_{\sigma R}} V\right),
$$

holds for any $z \in \mathbb{B}_{\tau R}$, where

$$
\rho(\sigma, \tau):=\frac{\log (\tau / \sigma)}{\log (1 / \sigma)} .
$$

This can be viewed as a "three-circle maximum principle".

Here we want to establish a kind of "three-circle minimum principle" which is dual in some sense to the previous one.

Define for $\sigma, \tau \in] 0,1[$ the following constant

$$
\nu(\sigma, \tau):=\frac{1}{\log \left(\frac{1+\sigma \tau}{\sigma+\tau}\right)} .
$$

Theorem 6.3 Let $\sigma, \tau \in] 0,1[$ be given real numbers. Then for any real number $\nu>\nu(\sigma, \tau)$ there exists constants $C=C(\sigma, \tau, \nu)>0$ and $0<\eta_{0}<$ 1 such that for any $R>0$, for any plurisubharmonic function $V$ on the euclidean ball $\mathbb{B}_{R}$ and for any $\left.\left.\alpha \in\right] 0,2\right]$ and any $\left.\eta \in\right] 0, \eta_{0}[$, the following lower bound

$$
V(z) \geq \sup _{\mathbb{B}_{\sigma R}} V+\nu \log (\eta / C)\left(\sup _{\mathbb{B}_{R}} V-\sup _{\mathbb{B}_{\sigma R}} V\right),
$$

holds for all $z \in \mathbb{B}_{\tau R}$ outside the union of a countable family of euclidean balls of radii $\left(r_{j}\right)$ not exeeding $\eta R$ and satisfying the estimate

$$
\sum_{j} r_{j}^{2 n-2+\alpha}<9^{n-1}(R e)^{2 n-2+\alpha} \eta^{\alpha} / \alpha .
$$

Observe that the theorem gives a precise uniform bound of the relative Hausdorff content of dimension $(2 n-2+\alpha)$ of the exceptionnal set where the lower estimate (6.1) does not hold. Proof: Since the inequality (6.1) and the condition (6.2) are invariant under any homothetic map, we can assume that $R=1$. Denote by $\mathcal{U}$ the class of plurisubharmonic functions on $\mathbb{B}$ such that $u \leq 1$ and $\max _{\mathbb{B}_{\sigma}} u \geq 0$. Then $\mathcal{U}$ is a compact class of plurisubharmonic functions. To apply Theorem 6.1 , we need to estimate 
the Lelong numbers of the class $\mathcal{U}$ on the compact ball $\overline{\mathbb{B}}_{\tau}$. Indeed, if $u \in \mathcal{U}$ and $z \in \overline{\mathbb{B}}_{\tau}$, then if $0<r<1$, we have

$$
\vartheta_{u}(z)=\vartheta_{u \circ \Phi_{z}}(0) \leq \frac{\sup _{\mathbb{B}} u \circ \Phi_{z}-\sup _{\mathbb{B}_{r}} u \circ \Phi_{z}}{\log (1 / r)} \leq \frac{1-\max _{\Phi_{z}\left(\mathbb{B}_{r}\right)} u}{\log (1 / r)} .
$$

Now it follows from a simple computation (see [30]) that for $z \in \mathbb{C}^{n}$ with $|z| \leq \tau$, the set $\Phi_{z}\left(\mathbb{B}_{r}\right)$ contains the ball $\mathbb{B}_{\sigma}$ precisely when $r=(\sigma+\tau) /$ $(1+\sigma \tau)$, which implies that $\max _{\Phi_{z}\left(\mathbb{B}_{r}\right)} u \geq 0$.

Then for any $u \in \mathcal{U}$ and any $z \in \overline{\mathbb{B}}_{\tau}$ we have

$$
\vartheta_{u}(z) \leq \nu(\sigma, \tau)
$$

This inequality was first obtained in [7].

Therefore from Theorem 6.1, it follows that for any $\nu>\nu(\sigma, \tau)$, there exists a constant $C>0$ and a real number $\left.\eta_{0} \in\right] 0,1[$ small enough, for any $0<\eta<\eta_{0}$, for any $\left.\left.\alpha \in\right] 0,2\right]$ and for any $u \in \mathcal{U}$, we get the following uniform lower bound

$$
u(z) \geq-\log (C / \eta),
$$

for any $z \in \mathbb{B}_{\tau}$ outside the union of a countable family of balls with radius $\left(r_{j}\right)$ less that $\eta$ and satistying the estimate $\sum_{j} r_{j}^{2 n-2+\alpha}<9^{n-1} \eta^{\alpha} / \alpha$.

Now let $V$ be an arbitrary non constant plurisubharmonic function on $\mathbb{B}$ so that $\sup _{\mathbb{B}} V>\sup _{\mathbb{B}_{\sigma}} V$. Then the following function

$$
u:=\frac{V-\sup _{\mathbb{B}_{\sigma}} V}{\sup _{\mathbb{B}} V-\sup _{\mathbb{B}_{\sigma}} V}
$$

belongs to the class $\mathcal{U}$. Therefore this function satisfies the lower bound (6.3) which yields the required lower bound for $V$.

As a consequence we give the following generalisation of the one variable minimum principle stated in section 2 .

Corollary 6.4 There exists a constant $C>1$ and a real number $0<\eta_{0}<1$ such that for any real number $0<\eta \leq \eta_{0}$ and any plurisubharmonic function $V$ is on any euclidean ball $\mathbb{B}_{\tilde{R}} \subset \mathbb{C}^{n}$, where $\tilde{R}:=2 e R$ and $R>0$ satisfying the condition $V(0)=0$, the following lower bound

$$
V(z) \geq-\log (C / \eta) \max _{\mathbb{B}_{\tilde{R}}} V
$$

holds for any $z \in \mathbb{B}_{R}$ outside the union of a countable family of balls of radii $\left(r_{j}\right)$ less than $\eta R$ which satisfy the following condition

$$
\sum_{j} r_{j}^{2 n-2+\alpha}<9^{n-1}(R e)^{2 n-2+\alpha} \eta^{\alpha} / \alpha
$$


Proof: It is enough to apply the last result with $\tilde{R}$ instead of $R, \tau=1$ / (2e) and $\sigma>0$ small enough so that $\nu(\sigma, \tau)<1$, which is possible since $\nu(0, \tau)=1 /(\log (2 e))<1$.

Let us give an asymptotic formulation of the last result which explains how the above three-circle minimum principle is dual to the three-circle maximum principle .

Let us define for $\varepsilon>0$ the $\left(h_{p}, \varepsilon\right)$-essentiel lower bound of a plurisubharmonic function $u$ on the ball $\mathbb{B}_{\tau R}$ as follows

$$
\mathcal{I}_{p, \varepsilon}\left(u, \mathbb{B}_{\tau R}\right):=\sup \left\{\inf _{\mathbb{B}_{\tau R} \backslash E} u ; E \subset \mathbb{B}_{\tau R}, h^{p *}(E) \leq \varepsilon\right\},
$$

where $h^{p *}$ is the exterior Hausdorff content or order $p$. Since $u$ may have poles, the real numbers $\mathcal{I}_{p, \varepsilon}\left(u, \mathbb{B}_{\tau R}\right)$ may decrease to $-\infty$ when $\varepsilon \downarrow 0$. The minimum principle above gives the rate of convergence to $-\infty$ of this minimum uniformly when the function $u$ is suitably normalized. More precisely, we deduce the following result.

Corollary 6.5 Let $\mathcal{U}$ the class of plurisubharmonic function on tha ball $\mathbb{B}_{R}$ such that $u \leq 1$ on $\mathbb{B}_{R}$ and $\max _{\sigma B} u=0$. Then for any $\left.\left.\alpha \in\right] 0,2\right]$, the following uniform asymptotic estimate

$$
\limsup _{\varepsilon \downarrow 0}\left(\sup _{u \in \mathcal{U}} \frac{\mathcal{I}_{p, \varepsilon}\left(u, \mathbb{B}_{\tau R}\right)}{\log \varepsilon}\right) \leq \frac{\nu(\sigma, \tau)}{\alpha}
$$

holds with $p=2 n-2+\alpha$.

\section{$7 \quad$ A minimum principle for quasi-plurisubharmonic functions}

Let $X$ a be compact Kähler manifold of dimension $n$ and $\omega$ a closed positive current on $X$ with bounded local potentials such that the volume $\operatorname{Vol}_{\omega}(X):=\int_{X} \omega^{n}>0$.

Let us fix adenote by $d$ the geodesic metric on $X$ et denote by $h^{p}$ the Hausdorff content of dimension $p$ on the metric space $(X, d)$.

Recall that a function $\varphi: X \longmapsto \mathbb{R} \cup\{-\infty\}$ is called an $\omega$-plurisubharmonic function on $X$ if $\varphi$ is upper semi-continuous on $X$ and satisfies the condition $d d^{c} \varphi+\omega \geq 0$ in the sense of currents on $X$. Therefore each point of $X$ has a small neighbourhood $U \subset X$ which is biholomorphic to a an euclidean ball in $\mathbb{C}^{n}$ so that $v_{U}:=\varphi+p_{U}$ is a plurisubharmonic function on a neighbourhood of $\bar{U}$, where $p_{U}$ is a local bounded potential for $\omega$ on a neighbourhood of $\bar{U}$ i.e. $d d^{c} p_{U}=\omega$ on a neighbourhood of $\bar{U}$. By compactness, $X$ can be covered by a finite number of such domains. Let us denote by $N=N(X)$ the minimum number of such domains necessary to cover $X$.

Observe that the Lelong numbers of an $\omega$-plurisubharmonic function $\varphi$ are well defined by the formula $\vartheta_{\varphi}(x)=\vartheta_{v}(x)$, where $v:=\varphi+p$ is 
plurisubharmonic on a neighbourhood of $x$ and $p$ is a bounded local potential of $\omega$ in a neighbourhood of $x$.

Let us define the maximal Lelong number of $(X, \omega)$ by the formula

$$
\vartheta(X, \omega):=\sup \left\{\vartheta_{\varphi}(x) ; \varphi \in P S H(X, \omega), x \in X\right\} .
$$

It is clear that $\vartheta(X, \omega)=\sup \left\{\vartheta_{\varphi}(x) ; \varphi \in \mathcal{P}_{0}, x \in X\right\}$, where $\mathcal{P}_{0}:=\{\varphi \in$ $\left.P S H(X, \omega) ; \sup _{X} \varphi=0\right\}$. Since $\mathcal{P}_{0}$ is compact fro the $L^{1}$ topology (see [17]), a standard compactness argument shows that $\vartheta(X, \omega)<+\infty$ (see [39]).

It's well known that if $X$ is a projective manifold and $\omega=\omega_{F S}$ is the Fubini-Study Kähler form on $X$ then $\vartheta(X, \omega)=\int_{X} \omega^{n}$, which is equal to the degree of algebraicity of $X$ (see [38]). We do not know whether this formula is true in the general case.

To state an analogue of Bernstein-Walsh inequalities which is the couterpart of the classical Hadamard-three circle maximum principle for the Lelong class $\mathcal{L}\left(\mathbb{C}^{n}\right)$, let us recall the definition of the global capacity of Borel sets in $(X, \omega)$ (see $[17])$.

For any Borel subset $K \subset X$, we define the $\omega$-capacity of $K$ in $X$ by

$$
T_{\omega}(K):=\exp \left(-\sup _{X} V_{K, \omega}\right),
$$

where $V_{K, \omega}$ is the extremal $\omega$-plurisubharmonic function associated to $K$ and defined as follows:

$$
V_{K, \omega}(x):=\sup \{\varphi(x) ; \varphi \in P S H(X, \omega)\}, x \in X .
$$

Then by [17], for any non pluripolar subset $K \subset X, T_{\omega}(K)>0$ is the best constant such that

$$
\varphi(x) \leq \sup _{K} \varphi-\log T_{\omega}(K), \forall \varphi \in P S H(X, \omega), \forall x \in X .
$$

Now we can state the minimum principle for $\omega$-plurisubharmonic functions analoguous the minimum principle for plurisubharmonic functions. Namely we obtain the following result.

Theorem 7.1 For any $\nu>\vartheta(X, \omega)$ there exists a constant $C=C(X, \omega, \nu)>$ 0 and a real number $\eta_{0}>0$ small enough such that for any real number $0<\alpha \leq 2$, any real number $0<\eta \leq \eta_{0}$ and any $\omega$-plurisubharmonic function $\varphi$ on $X$ the following lower bound

$$
\varphi(x) \geq \sup _{X} \varphi-\nu \log (C / \eta)
$$

holds for any $z \in X$ outside the union of a countable family of balls of radii $\left(r_{j}\right)$ satisfying the following condition

$$
\sum r_{j}^{2 n-2+\alpha}<N 9^{n-1} \eta^{\alpha} / \alpha .
$$


Proof: Take a covering of $X$ by $N=N(X)$ domains $\left(U_{i}\right)$ for which there are domains $\left(U^{\prime} i\right)$ biholomorphic to the unit ball of $\mathbb{C}^{n}$ with $U_{i} \Subset U_{i}^{\prime} \subset X$ for $1 \leq i \leq N$ and write $v_{i}:=\varphi_{i}+p_{i}$ where $p_{i}$ is a bounded local potential for $\omega$ on $U_{i}^{\prime}$.

We can apply the same method as in the proof of Theorem 6.1. Then from the fact that $p_{i}$ is a bounded plurisubharmonic function on $U_{i}^{\prime}$, we obtain for each $i$ constants $a_{i}>0, b_{i}, c_{i}>0$ such that the following lower bound

$$
\varphi(z) \geq a_{i} \int_{\partial U_{i}^{\prime}} \varphi d \sigma_{2 n-1}-\theta_{\varphi}(z, s) \log (3 / \eta)-b_{i} \mu_{\varphi}\left(U_{i}^{\prime}\right) \log (e / s)-c_{i},
$$

holds for all $z \in U_{i} \backslash E_{i}$, where $E_{i} \subset U_{i}$ is a Borel set with $h_{2 n-2+\alpha}^{\eta}\left(E_{i}\right)<$ $9^{n-1} \eta^{\alpha} / \alpha$. By compactness of the $\mathcal{P}_{0}:=\left\{\varphi \in P S H(X, \omega) ; \max _{X} \varphi\right\}=0$ (see [17]), it follows that $\int_{\partial U_{i}^{\prime}} \varphi d \sigma_{j}$ and $\mu_{\varphi}\left(U_{i}\right)$ are uniformly bounded for $\varphi \in \mathcal{P}_{0}$. Therefore taking $E:=\bigcup_{1 \leq i \leq N} E_{i}$, we obtain for a suitable constant $C>0$ the following lower bound

$$
\varphi(z) \geq-\theta_{\varphi}(z, s) \log (3 / \eta)-C \geq-\nu \log (3 / \eta)-C,
$$

for any $z \in X \backslash E$ and any $\varphi \in \mathcal{P}_{0}$. Since $h_{\eta}^{2 n-2+\alpha}(E) \leq \sum_{i} h_{2 n-2+\alpha}^{\eta}\left(E_{i}\right)<$ $N 9^{n-1} \eta^{\alpha} / \alpha$ and $\varphi-\max _{X} \varphi \in \mathcal{P}_{0}$ for any $\varphi \in P S H(X, \omega)$, we obtain the estimate of the theorem with an appropriate constant.

Now we want to prove the following comparison inequality.

Theorem 7.2 For any $0<\theta<\theta(X, \omega)$, there exists constant $C>0$ such that for any $0<\alpha \leq 2$ the following comparison inequality

$$
h^{2 n-2+\alpha}(K) \leq \frac{C}{\alpha} T_{\omega}(K)^{\alpha \theta},
$$

holds for ay Borel subset $K \subset X$.

Problem: Is the theorem still true with $\theta=\theta(X, \omega)$ ?

Aknowlegments: A lecture on a part of the preliminary version of this paper was presented in jully 16, 2005 in Rabat at the conference held in honour to my colleague and friend Bensalem Jennane. It's a pleasure for me to dedicate this work to him and to thank him for his valuable collaboration and his kind hospitality during all my numerous stays in Rabat. I whould also like to thank Abdelhak Azhari, Ahmed Sebbar and Alain Yger for interesting discussions on this subject and for providing me interesting references.

\section{References}

[1] H.Alexander, Projective capacity, Ann. of Math. Studies, Conference on Several Complex Variables, Princeton, 100 (1981), 3-27. 
[2] F.Amghad, Fonctions plurisousharmoniques à croissance logarthmique et potentiels logarithmiques dans $\mathbb{C}^{n}$, Diplôme des études supérieures de troisième cycle, Université Mohammed V, Rabat (1992).

[3] V.Avanissian, Fonctions plurisousharmoniques et fonctions doublement sousharmoniques, Ann. Sci. Ecole Norm. Sup., 78 (1961), 101-161.

[4] V.Avanissian, Quelques applications de la méthode des "boules d'exclusion" dans $\mathbb{C}^{n}$, Izv. Akad. Nauk Armjan. SSSR Ser. Mat., 8 (1973), 306-320.

[5] E.Bedford and B.A.Taylor, A new capacity for plurisubharmonic functions, Acta Math., 149 (1982), 1-40 .

[6] E.Bedford and B.A.Taylor, Plurisubharmonic functions with logarithmic singularities, Ann. Inst. Fourier, Grenoble, 38-4 (1998), 133-171.

[7] S.Benelkourchi and B.Jennane, Intégrabilité uniforme semiglobale d'une classe de fonctions plurisousharmoniques, C.R.Math.Acad.Sci.Paris, 337-4 (2003), 239-242.

[8] S.Benelkourchi, B.Jennane and A.Zeriahi, Polya's inequalities, global integrability and the size of plurisubharmonic lemniscates, Ark. for Math., 43-1 (2005), 85-112.

[9] C.A.Berenstein and B.A.Taylor, Interpolation problems in $\mathbb{C}^{n}$ with applications to Harmonic Analysis, J. Analyse Math., 38 (1980), 188-254.

[10] H. Cartan, Sur les systèmes de fonctions holomorphes à variétés linéaires et leurs applications, Ann. Sci. Ecole Norm. Sup., 45 (1928), 255-346.

[11] U.Cegrell, Pluricomplex energy, Acta Math., 180 (1998), 187-217.

[12] U.Cegrell, The general definition of the Monge-Ampère operator, Ann. Inst. Fourier, Grenoble, 54, 1 (2004), 159-179.

[13] A.Cuyt, K.Driver, D.S.Lubinsky, On the size of lemniscates of polynomials in one and several variables, Proc. Amer. Math. Soc., 1247(1996), 2123-2136.

[14] J-P. Demailly, Potential theory in several complex variables, Ecole d'été d'Analyse complexe du CIMPA, Nice, 1989.

[15] J-P.Demailly, Monge-Ampère operators, Lelong numbers and intersection theory, Proceedings of the $10^{\text {th }}$ Trento Conference on Complex Analysis and Geometry. 
[16] L.Ehrenpreis, Solution of some problems of division. Part IV: invertible and elleptic operators, Amer. J. Math., 82 (1960), 522-588.

[17] V.Guedj and A.Zeriahi, Intrinsic capacities on compact Kähler manifolds, J. Geometric Analysis,15-4 (2005), 607-639.

[18] G.A. Gonchar, A local condition of single-valuedness of analytic functions, Mat. Sbornik, 89 (1972, 151-167.

[19] L.Hörmander, Notions of convexity , Progress in Mathematics, Birkhäuser, Boston, 1994.

[20] C.O.Kiselman, Densité des fonctions plurisousharmoniques, Bull. Soc. Math. de France , 107 (1979), 295-304.

[21] M.Klimek, Pluripotential theory, Oxford University Press, London, 1991.

[22] , S.Kolodziej, The logarithmic capacity in $\mathbb{C}^{n}$, Ann. Polon. Math.,48 (1988), 253-267.

[23] S.Kolodziej, The complex Monge-Ampère equation and Pluripotential Theory, Memoirs of the AMS, Vol. 178, No 840, 2005.

[24] N.S.Landkof, Foundations of modern potential theory, Springer, Berlin, 1972.

[25] P.Lelong, Fonctions plurisousharmoniques et formes différentielle positives, Gordon et Breach, New-York et Dunod, Paris, 1969.

[26] B.Ya.Levin, Lectures on entire functions, Translations of Mathematical Monographs, AMS, Vol.150, 1996.

[27] N.Levenberg and B.A.Taylor, Comparison of capacities in $\mathbb{C}^{n}$, Proceedings of Complex Analysis colloqium, Toulouse 1983, LNM number 1094, p. $162-172$.

[28] D.S.Lubinsky, Small values of polynomials: Cartan, Pólya and others, J. of Inequal. and Appl., 1 (1997), 199-222.

[29] P.Mattila, Geometry of Sets and Measures in Euclidean Spaces, Cambridge Studies in Advanced Mathematics, Cambridge University Press, 1995.

[30] H.Milloux, Sur une extension d'un théorème de P.Boutroux-H.Cartan, Bull. Soc. Math. de France, 65 (1937), 65-75.

[31] W.Rudin, Function theory in the unit ball of $\mathbb{C}^{n}$, Springer Verlag, New York, Heidelgerg, Berlin , 1980. 
[32] J.Siciak, Extremal plurisubharmonic functions in $\mathbb{C}^{N}$, Ann. Polon. Math., 39 (1981), 175-211.

[33] J.Siciak, Extremal plurisubharmonic functions and capacities in $\mathbb{C}^{N}$, Sophia Kokyuroku in Math., Sofia University, Tokyo, Vol. 14, 1982.

[34] M.Stoll, Invariant Potential Theory, London Math. Soc. Lecture Notes 199, Cambridge University Press 1994.

[35] A.Yger, Fonctions holomorphes et distributions, Collection Ellipses, 2001.

[36] V.P.Zahariuta, Extremal plurisubharmonic functions, orthogonal polynomials and Bernstein-Walsh theorem for analytic functions of several complex variables, Ann. Polon. Math., 33 (1976), 137-148.

[37] A.Zeriahi, Capacité, constante de Chebysheff et polynômes orthogonaux associés à un compact de $\mathbb{C}^{n}$, Bull. Sc. Math., 109-3 (1985), 325-335.

[38] A.Zeriahi, A criterion of algebraicity for Lelong classes and analytic sets, Acta Math., 184 (2000), 113-143.

[39] A.Zeriahi, Volume and capacity for sublevel sets of plurisubharmonic functions in a Lelong class, Indiana Univ. Math. J., 50-1 (2001), 671702 .

[40] A.Zeriahi, The size of plurisubharmonic lemniscates in terms of Hausdorff-Riesz measures and capacities, Proc. London Math. Soc., 893 (2004), 104-122.

Université Paul Sabatier - Toulouse 3,

Laboratoire de Mathématiques Emile Picard,

118 route de Narbonne,

31062 Toulouse Cedex, France

e-mail: zeriahi @ math.ups-tlse.fr 